\title{
Molecular Imaging and Precision Medicine in Breast Cancer
}

\author{
Amy V. Chudgar, MD and David A. Mankoff, MD, PhD \\ Department of Radiology, Division of Nuclear Medicine \\ University of Pennsylvania, Philadelphia, PA 19102
}

Corresponding Author:

David Mankoff, MD, PhD

Donner 116

Division of Nuclear Medicine

Department of Radiology

Hospital of the University of Pennsylvania

3400 Spruce Street

Philadelphia, PA 19104

Phone: 215.615 .3687

Fax: 215.349.5843

E-mail: david.mankoff@uphs.upenn.edu

Amy Chudgar, MD

Division of Nuclear Medicine

Department of Radiology

Hospital of the University of Pennsylvania

3400 Spruce Street

Philadelphia, PA 19104

Phone: 706.373 .8479

Fax: 215.349.5843

Email: amy.chudgar@uphs.upenn.edu

The authors have nothing to disclose.

\section{Acknowledgements}

This work was supported in part by Susan G. Komen SAC130060 and Department of Energy DE-SE0012476 grants. 


\section{Synopsis}

Precision medicine, namely basing treatment approaches on patient traits and specific molecular features of disease processes, has an increasingly important role in the management of breast cancer patients as targeted therapies continue to improve. PET imaging offers noninvasive information that is complementary to traditional tissue biomarkers, including information about tumor burden, tumor metabolism, receptor status and proliferation. Several PET agents that image breast cancer receptors can visually demonstrate the extent and heterogeneity of receptor positive disease and can help predict which tumors are likely to respond to targeted treatments. Furthermore, PET agents can aid in drug development by demonstrating successful drug binding to target and early response of specific therapies. Molecular imaging may also provide a key indicator of outcome at later time points in breast cancer treatment. This review will present an overview of applications of PET imaging in the targeted treatment of breast cancer.

\section{$\underline{\text { Key Words }}$}

Breast cancer, precision medicine, biomarkers, fluorodeoxyglucose (FDG), $16 \alpha-$ $\left[{ }^{18} \mathrm{~F}\right]$ fluoro-17 $\beta$-estradiol (FES)

\section{Key Points:}

- Molecular imaging can serve as a biomarker in breast cancer and provide a complementary, non-invasive global evaluation of the disease process and information to guide treatment. 
- Imaging of estrogen, progesterone, and human epidermal growth factor (HER2) receptors may help predict which patients are the most likely to endocrine or HER2 targeted therapy.

- PET imaging can be used in breast cancer drug development, drug dose optimization, and assessment of early response to therapy.

- Molecular imaging may also provide a key indicator of outcome at later time points in breast cancer treatment. 


\section{Introduction}

Breast cancer is the most common malignancy in women, with an estimated 234,000 new cases diagnosed in 2015 and approximately 5-9\% of patients with metastatic disease at presentation (1). Biomarkers have become increasingly important in breast cancer treatment for prognosis and for guiding treatment decisions (2). Precision medicine involves the use of biomarkers to create individualized and targeted treatments (2). In addition to traditional tissue-based biomarkers, molecular imaging can also serve as a biomarker and provides a complementary, non-invasive global evaluation of the disease process and can provide information to guide treatment, such as prognosis, drug pharmacodynamics and response to treatment.

Precision medicine in breast cancer has traditionally been based on results of tissue sampling and assay of the primary breast tumor and metastatic sites - for example, assaying the estrogen receptor (ER) to select patients who may benefit from endocrine therapy (3). However there are limitations of biopsy, such as sampling error and invasiveness. Biopsy of the primary neoplasm is not always representative of the entire tumor because of intratumor heterogeneity (4). Tissue sampling of metastatic lesions is performed when possible but certain metastatic sites, such as bone and brain, are not always amenable to biopsy and receptor status is often inferred from the primary tumor. However receptor status can vary from the primary tumor and metastatic lesions in 25$40 \%$ of patients (5). Even when biopsy of metastatic sites is possible, tissue sampling of metastatic lesions is not always representative of the entirety of the disease because of tumor heterogeneity (4). Monitoring response to targeted treatment to evaluate for changes in the tumor biology would require resampling of primary and metastatic lesions 
and is invasive and often painful. Furthermore there are risks associated with biopsy, such as bleeding and infection.

Imaging of biomarkers through positron emission tomography/computed tomography (PET/CT) offers a complementary, non-invasive method to obtain biological information regarding breast cancer, including tumor burden, tumor metabolic activity, receptor status and proliferation index. This review focuses on the use of molecular imaging as a biomarker in breast cancer precision medicine, with a focus on: 1) imaging as a prognostic biomarker, 2) imaging as a predictive biomarker, 3) imaging to evaluate drug pharmacodynamics, 4) imaging to determine early response to therapy, and 5) imaging to predict biological response (Table 1). Through example of the use of molecular imaging to accomplish each of these tasks, we will highlight several different PET radiopharmaceuticals used either clinically or in clinical trials and describe recent clinical studies that demonstrate the impact and promising future role of imaging in precision medicine.

\section{Prognostic Factors and Breast Cancer}

Prognostic factors help to distinguish which tumors are most likely to progress to tumor spread and death. Some examples of prognostic factors in breast cancer are ER, PR, and HER2 receptor status, proliferation index, and Oncotype DX 21-gene panel (6-8). Most of these prognostic indicators have traditionally been evaluated through tissue assay, typically via immunohistochemistry. An important prognostic factor for breast cancer is the size of the tumor and its extent of spread - i.e., staging. As such, imaging serves to help assess breast cancer prognosis by determining the stage of breast cancer. 
The National Comprehensive Cancer Network (NCCN) guidelines currently lists fluorodeoxyglucose (FDG)-PET/CT as a consideration in staging clinical stage III or higher breast cancer (9). FDG-PET/CT has been shown as a tool for prognostic stratification through staging $(10,11)$. Cochet et al prospectively evaluated 142 patients with at least grade T2 tumor, comparing conventional imaging against FDG-PET/CT and found FDGPET/CT provided stronger prognostic stratification of progression free survival compared to conventional imaging $(\mathrm{p}<0.0001)$ and FDG-PET/CT was an independent predictor of recurrence or progression $(\mathrm{p}<0.001)(11)$. Groheux et al showed in a prospective study of 189 patients with clinical stage IIB disease, 47 patients with M1 disease discovered on FDG-PET/CT had statistically significant shorter three-year disease specific survival, $57 \%$ vs $88 \%(\mathrm{p}<.001$ ) (10). In this same group of patients, M1-disease and triple-negative phenotypes were found to be two statistically significant independent prognostic variables (10).

Additionally, several studies have also demonstrated that the level of FDG uptake in the primary breast tumor, typically measured as the standardized uptake value (SUV), and in metastases serves as a prognostic factor itself and is indicative of histological grade, triple negative status, and high proliferation index (Ki-67 expression) (11-16). Although FDG-PET/CT is used for diagnosis and staging, the intensity of FDG uptake on PET/CT in the primary tumor and in metastatic lesions also serves as an important prognostic factor (11-14). As an analog of glucose, FDG uptake is a measures glycolytic rate. Studies using FDG-PET/CT have shown that elevated glycolysis is associated with a number of aggressive cancer features, such as proliferation and enhanced survival (17). High FDG uptake in the primary breast tumor has been associated with poor prognostic 
factors including high histological grade, triple negative status, or p53 mutation $(17,18)$. In the above-mentioned study, Cochet et al found that higher FDG uptake was associated with aggressive features, with a 3:1 ratio of baseline FDG uptake between triple negative and luminal A tumors (11). Koolen et al found in 203 primary stage II or III breast cancer patients evaluated with FDG-PET/CT, a higher SUV value was associated with aggressive features, such as distant metastases at time of staging, triple negative tumors, and grade 3 tumors (12).

Tumor as estimated by tissue sampling and the by Ki-67 immunochemistry index also provides prognostic information. Several studies have demonstrated a correlation between high proliferation index (Ki-67 expression) and higher FDG uptake $(12,15)$. Furthermore, invasive breast tumors with higher grades demonstrated higher FDG uptake compared to lower grade tumors $(14,15)$. Triple negative breast cancer patients typically have higher FDG avidity $(14,16)$.

\section{$\underline{\text { Precision Medicine and Predictive Factors }}$}

Predictive factors indicate whether or not a specific treatment is likely to be beneficial to the patient, for example, whether a patient may benefit from endocrine therapy. Predictive biomarkers such as ER, PR, and HER2 based upon tissue assays are widely used to direct breast cancer systemic therapy (4). However the results may not be reflective of the entire burden of disease due to intratumoral and metastatic heterogeneity. Imaging biomarkers offer an approach that is complementary to tissue sampling to help guide treatment decisions. 
Approximately $70 \%$ of breast cancers express estrogen receptors and treatment with endocrine therapy has been one of the key factors improving breast cancer mortality. Endocrine therapy is considered the preferred first line treatment and is the most effective treatment for metastatic ER+ breast cancer, but only 50 to $75 \%$ of ER+ breast cancer patients will respond to first line endocrine therapy (19). Beyond first line therapy, response to endocrine therapy decreases to $25 \%$ due to resistance through various mechanisms (19).

The PET radiopharmaceutical 16 $\alpha-\left[{ }^{18} \mathrm{~F}\right]$ fluoro-17 $\beta$-estradiol (FES), an estradiol analog, is the most researched radioligand for imaging the estrogen receptor. Measurement of SUV on FES-PET/CT has been shown to correlate with ER expression when compared to immunohistochemistry $(20,21)$. Several studies have addressed FES as a predictive biomarker. Prior FES studies have shown that FES-PET/CT, using both qualitative and quantitative measures can identify which patients are most likely to benefit from endocrine therapy (20,22-24). For example, Dehdashti et al found in 11 patients, a baseline FES SUV in responders was $\geq 2.2$ in patients who responded to Tamoxifen therapy at 2 months and $\leq 1.7$ in the nonresponders (22). Mortimer et al found in $40 \mathrm{ER}+$ breast cancer patients images with FES-PET/CT and FDG-PET/CT before and 7-10 days after Tamoxifen therapy, baseline SUV of FES uptake in responders was 4.3 +/2.4 compared to non-responders with SUV of $1.8+/-1.3 ; p=0.0007$ (25). Linden et al found in 47 pretreated patients with ER+ tumors, 0 of 15 patients with baseline SUV $<1.5$ responded to endocrine therapy versus 11 out of 32 patients with $\operatorname{SUV}>1.5(\mathrm{p}<0.01)$ (Figure 1) (24). In assessing studies published across heterogeneous populations, the FES SUV threshold of 1.5 retained predictive value, but with imperfect sensitivity or 
specificity for predicting response endocrine to therapy (26). Further studies are necessary to determine the sensitivity and specificity of baseline FES-PET/CT SUV value to predict response to endocrine therapy and identify optimal thresholds. This question is actively being addressed in a phase II trial enrolling patients with ER+ metastatic breast cancer receiving first line endocrine therapy (NCT02398773) (27).

Progesterone receptor, an estrogen-regulated gene, also serves as a predictive biomarker and is routinely evaluated in immunohistochemical assays. The presence of expression of PR with expression of ER increases the likelihood to respond to endocrine therapy (7). 21-(18)F-fluoro-16 $\alpha, 17 \alpha-[(\mathrm{R})-(1 '-\alpha$-furylmethylidene)dioxy]-19-norpregn-4ene-3,20-dione (FFNP) is the radioligand with high affinity and selectivity for the PR and shows the most promise for PR imaging $(28,29)$. Preclinical breast models have demonstrated decreased uptake of FFNP predicts tumors that will respond to fulvestrant and estrogen-deprivation therapy. (30). Although less research has been performed on FFNP-PET/CT as a predictive biomarker, a current clinical trial (NCT02455453) is addressing this topic (31). In this trial, FFNP-PET/CT scans are being performed in ER+ postmenopausal breast cancer patients before and after the administration of estradiol for one day (estrogen challenge) to determine look at changes in in FFNP maximum SUV measurement.

Human epidermal growth factor receptor type 2 (HER2) is overexpressed in approximately $15-25 \%$ of invasive breast cancer and is associated with aggressive disease (32-35). HER2 is routinely assayed through immunohistochemistry or fluorescence in situ hybridization to determine which patients may benefit from HER2 directed therapy. However an estimated $50 \%$ of patients with overexpression of HER2 
breast cancer do not respond to HER2 directed therapy (36). The Neoadjuvant Lapatinib and/or Trastuzumab Treatment Optimisation (NEO-ALTTO) study enrolled 455 women with HER-2 positive breast cancer, of which 77 patients underwent serial FDG-PET/CTs and found that early FDG-PET/CT was able to identify patients more likely to have complete response to neoadjuvant trastuzumab and/or lapatinib with paclitaxel (37).

Directly imaging HER2 disease has been studied to visualize the tumor burden of HER2+ disease (38). Several imaging agents have been created to bind to the HER2 receptor and examples of the positron emitting radionuclides are ${ }^{64} \mathrm{Cu}$-trastuzumab, ${ }^{64} \mathrm{Cu}-$ DOTA-Z ${ }_{\text {HER2:477, }}{ }^{68} \mathrm{Ga}$-trastuzumab F(ab' b fragments, ${ }^{68} \mathrm{Ga}-\mathrm{ABY}-002$, and ${ }^{89} \mathrm{Zr}-$ trastuzumab $(39,40)$. The ZEPHIR study was the first large prospective trial evaluating ${ }^{89} \mathrm{Zr}$-trastuzumab-PET/CT (HER2-PET/CT) as a predictive biomarker for advanced HER2+ breast cancer patients for response to Trastuzumab emtansine (T-DM1), an antibody-drug conjugate to target HER2 receptor in patients who progress after prior line of trastuzumab-based therapy (Figure 2) (41). They found in 56 patients with advanced HER2 positive breast cancer, pretreatment imaging with HER2-PET/CT and imaging with FDG-PET/CT after 1 month demonstrated a high negative predictive value when compared to RECIST1.1 (88\%) (41). There are several prospective clinical trials underway to evaluate the clinical utility of HER2 molecular imaging, including 1 study at Institut Jules Bordet (NCT01420146) using ${ }^{89} \mathrm{Zr}$-Trastuzumab in HER2+ metastatic breast cancer correlated to FDG-PET/CT and two studies at the University Medical Center Groningen (NCT01832051 and NCT01957332) (42-44).

\section{Using molecular imaging to evaluate drug binding to target}


Molecular imaging can be used as a tool to evaluate binding of drug to target in the development of new drugs. For example, FES-PET/CT has been shown as an effective tool to assess endocrine therapy on ER binding (45). A retrospective study of 30 patients with metastatic breast cancer (predominately bone dominant) measured FES uptake before and after endocrine-targeted treatments, including aromatase inhibitors, tamoxifen, and fulvestrant (45). They found that estrogen blocking therapies (tamoxifen, fulvestrant) decreased FES binding over serial PET/CTs compared to aromatase inhibitors, demonstrating that FES-PET/CT might be a valuable clinical tool to visualize activity of endocrine therapy (45). The authors also noted differences in the ER blockade between the ER blocking agents tamoxifen and fulvestrant and hypothesized that the incomplete ER blockade in patients receiving fulvestrant was due to inadequate dosing of this medication (loading dose of $500 \mathrm{mg}$ followed by $250 \mathrm{mg}$ at 2 weeks $\mathrm{x} 2$ ) (45). This hypothesis was further supported by clinical studies showing increased efficacy of fulvestrant at higher doses $(46,47)$. A prospective study studied the efficacy of a higher dose of fulvestrant (500 mg) to determine if this dose was sufficient for complete ER blockade using FES-PET/CT (48). 38\% of patients treated with fulvestrant (6 out of 16) were found to residual FES uptake, which was associated with early clinical disease progression (48). These studies demonstrate that FES-PET/CT can be used for determining optimal ER blockade for drug development and optimization of therapeutic dose(49).

Another example of using FES-PET/CT in the evaluation of new drug development is evaluating ARN-810, a newer endocrine directed therapy to treat advanced ER+ breast cancer by blocking and degrading ER $(50,51)$. A recent multicenter, Phase I clinical trial 
evaluated targeting of ER by ARN-810 in 30 patients with advanced or metastatic ER+ breast cancer. Baseline and subsequent FES PET scans after initiation of ARN-810 demonstrated come to near complete (>90\%) suppression of FES uptake to background levels, indicating successful drug target binding (50).

HER2 imaging agents have been used in the evaluation of drug binding of HER2 directed therapies (8). For example, ${ }^{89} \mathrm{Za}$-trastuzumab and ${ }^{89} \mathrm{Zr}$-bevacizumab PET scans were used for quantitative assessment in the evaluation of NVP-AUY922, a HER2 targeted therapy that inhibits HSP90, a molecular chaperone with client proteins that play a role in metastatic breast cancer through HER2, hypoxia-inducible factor-1 $\alpha$, and ER (52). ${ }^{89} \mathrm{Za}-$ trastuzumab PET was found to positively correlate to tumor size after 3 weeks of HSP90 inhibitor treatment, indicating that PET probes such as ${ }^{89} \mathrm{Za}$-trastuzumab may be used for evaluation of HER2 targeted therapies. (52).

\section{Assessment of early response (pharmacodynamics)}

A pharmacodynamic response indicates some action of the drug on its target and provides an indication of efficacy in targeted cancer therapy. A pharmacodynamic response can indicate some likelihood of subsequent response to therapy, and perhaps more importantly, a lack of a pharmacodynamic response often indicates little chance of therapeutic success. Pharmacodynamics may also be used to determine the optimal dosing for therapy (6). Clinically, it may be difficult to infer the early impact of many targeted drugs, particularly those with a cytostatic rather than cytotoxic effect.

One example of an early pharmacodynamic effect can be seen in ER-targeted drugs with an agonist action. A "clinical flare reaction" is a clinical response in patients 2 weeks 
after initiating endocrine therapy with drugs with transient agonist effect, such as tamoxifen, characterized by pain in osseous metastases and increase in size of soft tissue metastases and is predictive of response to endocrine treatment (25). However this response is not always seen or recognized and is furthermore difficult to differentiate from disease progression clinically when seen at later time points (23). Several studies have demonstrated that a metabolic flare reaction may be detected through FDG-PET/CT imaging, which is predictive of response to endocrine therapy $(23,25,53)$. For example, Mortimer et al studied FDG-PET/CT before and after treatment with tamoxifen in postmenopausal ER+ breast cancer patients and found an increase in tumor FDG uptake after tamoxifen in 20 out of 21 responders versus no significant change in tumor FDG uptake in the 19 nonresponders $(p=0.0002)(25)$. A clinical flare reaction was seen in only 5 out of the 21 responders (25). Similarly, Dehadasti et al found that a metabolic flare reaction induced by an estradiol challenge could be detected as a significantly higher mean percent change in SUV for responders compared to non-responders on FDGPET/CT, and patients with a metabolic flare who were subsequently treated with endocrine therapy had significantly longer overall survival compared to those without a metabolic flare (23). To further expand on these results, Kurland et al studied the metabolic flare reaction in patients treated with aromatase inhibitors, which decrease circulating estradiol levels, with correlation to proliferation index (Ki-67) assays (53). They found a decrease in FDG SUV values over a two week course of aromatase inhibitor therapy, which corresponded to the lower post-treatment Ki-67 (53). These studies demonstrate that the presence of a metabolic flare response indicates which patients are more likely to respond to endocrine treatment. 
Tumor proliferation is most commonly performed by measuring Ki-67 labeling index through immunohistochemistry (54). 3'deoxy-3'-18F-fluorothymidine (FLT), a thymidine analog, is the most widely used proliferation probe that is dependent on the activity of thymidine kinase-1 (TK-1) and correlates with Ki-67 expression(55-57). An early decline in cellular proliferation assayed by Ki-67 an serial tissue biopsy has been to shown to provide an early indication of successful therapy for both chemotherapy and endocrine therapy, as soon as 1-2 weeks after starting treatment $(58,59)$. Similar findings have been seen using serial FLT-PET/CTs to measure early response to therapy in breast and other cancers $(60,61)$. A recent multi-center trial performed in the US has confirmed their early single-center findings (Figure 3) (62).

FLT PET/CT can also be used to evaluate the pharmacodynamics of drugs impacting components of the thymidine metabolic pathway. For example, thymidylate synthase (TS) inhibitor drugs, such as capecitabine, inhibit the de novo thymidine synthesis pathway and have their greatest impact in tumors that rely on this pathway, versus the external (salavage) pathway traced by FLT (63). Interruption of the de novo pathway by TS-targeted drugs can cause a transient increase in thymidine flux through the external pathway, which can be detected by PET proliferation imaging. In a novel study, tumor FLT uptake was found to be increased in patients after treatment with capecitabine, indicating a transient increase in external pathway thymidine flux with TS inhibition measured by imaging, and a potential method to measure the pharmacodynamics of thymidylate synthase inhibitors (63).

Another target for imaging proliferation is the sigma-2 receptor, a biomarker of tumor cells that is overexpressed in proliferating tumors (64-66). The most promising 
sigma-2 receptor radioligand is N-(4-(6,7-dimethoxy-3,4-dihydroisoquinolin-2(1H)yl)butyl)-2-(2-(18)F-fluoroethoxy)-5-methylbenzamide (18F-ISO-1), which has been found to correlate with laboratory measures of proliferation status in mouse mammary tumor model and with Ki-67 expression in 13 breast cancer patients $(67,68)$. This imaging probe shows promise as a predictive biomarker and may be complementary to FLT-PET/CT in the evaluation of cell cycle-targeted therapies, such as selective cyclin dependent kinase inhibitors (69).

Many other PET radiotracers have been studied in breast cancer drug pharmacodynamics, including ${ }^{11}[\mathrm{C}]$ choline and L-methyl-11 C-methionine $\left.{ }^{11} \mathrm{C}-\mathrm{MET}\right)(54)$. ${ }^{11}[\mathrm{C}]$ choline has greater uptake in tumor cells due to increased intracellular choline kinase activity in tumor cells (54). ${ }^{11}[\mathrm{C}]$ choline PET uptake decreased over serial scans in patients treated with trastuzumab, indicating that his radiotracer can be used for monitoring response to therapy (70). L-methyl-11 C-methionine $\left({ }^{11} \mathrm{C}-\mathrm{MET}\right)$ is an amino acid used in PET, and a small number of cases were found to have decreased ${ }^{11} \mathrm{C}$-MET uptake after treatment with endocrine therapy or chemotherapy (71-73). Numerous others have a potential important role in the development and optimization of targeted therapies $(7,54)$.

\section{Biological response: How does imaging based response predict outcome?}

In addition to measuring early response to treatment, molecular imaging may also provide a key indicator of outcome at later time points in breast cancer treatment. Molecular imaging may prove information complementary to size-based measures of response, particularly for disease sites that are difficult to evaluate by anatomic imaging. 
One primary example of using PET imaging to predict outcome is in bone dominant breast cancer, a site of disease that has been difficult to evaluate by standard imaging (Figure 4) (74). In bone dominant metastatic breast cancer, FDG-PET/CT has been demonstrated to demonstrate overall time to progression and overall survival (75-78). In a retrospective study of 253 patients with metastatic breast cancer, there was a strong correlation of maximum SUV of bone metastases on baseline FDG-PET/CT and overall survival (75). Although not statistically significant, the presence of liver, nodal and pulmonary metastases were found to have a greater risk of death (75). A retrospective study of 28 patients undergoing treatment demonstrated that changes in serial FDGPET/CT scans may predict time-to-progression and time to first skeletal-related event in bone-dominant metastatic breast cancer (76). A retrospective study of 102 women with metastatic breast cancer, a decrease in metabolic activity and increase in CT attenuation of osseous metastases after treatment was found as an independent predictor of response duration (77). A retrospective study of 35 patients with 146 identified osseous lesions, FDG uptake was found to correlate with tumor activity independent of the morphologic characteristics (78). Prospective studies are ongoing to confirm these retrospective studies.

Molecular imaging can also provide a robust indication of the impact of therapy in the neo-adjuvant setting, and measures of molecular imaging probes post-therapy have been shown to predict subsequent relapse and survival in a number of settings. Emmering and colleagues showed that the presence or absence of uptake post neoadjuvant chemotherapy predicted the likelihood of relapse (79), while Dunnwald showed that the change in quantitative FDG uptake measures mid-way through therapy 
predicted relapse-free and overall survival (80). Another study by Dunnwald et al (81) also indicated that measures of blood flow from [150]-water PET predicted disease-free and overall survival , providing incremental value to established prognostic factors, echoing earlier work showing the ability of serial imaging with [99m $\mathrm{Tc}]$-sestamibi to predict similar measures (82). These examples indicate the ability of molecular imaging to provide biologically-based measures of response that are highly predictive of key downstream outcomes such as relapse and survival.

\section{Conclusion}

The role of PET in breast cancer continues to evolve with broader applications in diagnostics and therapeutics in metastatic breast cancer patients. As precision medicine leads to the development of further targeted therapies, PET imaging with new radiotracers provides a complimentary and noninvasive method for prognostic and predictive information about tumor burden, tumor metabolic activity, receptor status and proliferation index. Furthermore, with the development and evolution of targeted treatments, PET imaging offers a method to assess drug binding to the target and optimization of drug dosage and has the potential role for an increasingly important tool in clinical trials. As we have reviewed, molecular imaging has shown an important role in the assessment of breast cancer tumor biology, disease burden, and drug development and offers great promise in the future of breast cancer management.

\section{Acknowledgements}


This work was supported in part by Susan G. Komen SAC130060 and Department of Energy DE-SE0012476 grants. 
Table 1: Sample applications of molecular imaging in breast cancer

\begin{tabular}{|c|c|c|}
\hline & Definition & Sample Applications \\
\hline $\begin{array}{l}\text { Prognostic } \\
\text { Factors }\end{array}$ & $\begin{array}{l}\text { Indicate which } \\
\text { tumors are most } \\
\text { likely to progress } \\
\text { to tumor spread } \\
\text { and death }\end{array}$ & $\begin{array}{l}\text {-FDG-PET/CT for prognostic } \\
\text { stratification through staging }(10,11) \\
\text {-FDG-PET/CT SUV measurement in } \\
\text { primary tumor for histological grade, } \\
\text { triple negative status, proliferation } \\
\text { index }(11-16)\end{array}$ \\
\hline $\begin{array}{l}\text { Predictive } \\
\text { Factors }\end{array}$ & $\begin{array}{l}\text { Whether or not } \\
\text { treatment is likely } \\
\text { to benefit the } \\
\text { patient. }\end{array}$ & $\begin{array}{l}\text {-FES-PET/CT for evaluation of ER } \\
\text { expression to predict response to } \\
\text { endocrine therapy }(20,22-26) \\
\text {-FFNP-PET/CT for evaluation of PR } \\
\text { expression to predict response to } \\
\text { endocrine therapy (28-30) } \\
\text {-89Zr-trastuzumab-PET/CT for HER2 } \\
\text { imaging to predict response to } \\
\text { trastuzumab-based therapy (41) }\end{array}$ \\
\hline $\begin{array}{l}\text { Drug } \\
\text { Binding to } \\
\text { Target }\end{array}$ & $\begin{array}{l}\text { Evaluate binding } \\
\text { of drug to target in } \\
\text { the development } \\
\text { of new drugs. }\end{array}$ & $\begin{array}{l}\text {-FES-PET/CT for evaluation of } \\
\text { aromatase inhibitors, tamoxifen, and } \\
\text { fulvestrant (45) } \\
\text {-FES-PET/CT for optimizing dose of } \\
\text { fulvestrant (48) }\end{array}$ \\
\hline
\end{tabular}




\begin{tabular}{|c|c|c|}
\hline & & $\begin{array}{l}\text {-FES-PET/CT in the evaluation of } \\
\text { ARN-810 (50) } \\
\text {-89 Zr-trastuzumab-PET/CT in the } \\
\text { evaluation of HSP90 inhibitors (52) }\end{array}$ \\
\hline $\begin{array}{l}\text { Assessment } \\
\text { of Early } \\
\text { Response }\end{array}$ & $\begin{array}{l}\text { Indicates some } \\
\text { action of the drug } \\
\text { on its target and } \\
\text { provides an } \\
\text { indication of } \\
\text { efficacy in targeted } \\
\text { cancer therapy }\end{array}$ & $\begin{array}{l}\text {-FDG-PET/CT showing a "metabolic } \\
\text { flare reaction" after treatment with } \\
\text { tamoxifen or with estradiol challenge } \\
(23,25,53) \\
\text {-FLT-PET/CT for response to } \\
\text { chemotherapy and endocrine } \\
\text { therapy }(58,59)\end{array}$ \\
\hline $\begin{array}{l}\text { Biological } \\
\text { Response }\end{array}$ & $\begin{array}{l}\text { Indicator of } \\
\text { outcome at later } \\
\text { time points in } \\
\text { treatment }\end{array}$ & $\begin{array}{l}\text {-FDG-PET/CT to predict outcome is } \\
\text { in bone dominant breast cancer (75- } \\
78 \text { ) } \\
\text {-FDG-PET/CT in the neo-adjuvant } \\
\text { setting and post-therapy to predict } \\
\text { subsequent relapse and survival (79- } \\
82 \text { ) }\end{array}$ \\
\hline
\end{tabular}

Figure 1: FES- and FDG- PET/CT in two patients demonstrating FES as a predictive marker for response to endocrine therapy. Row A demonstrates a patient with multiple FES- and 
FDG-avid osseous metastases and post-treatment FDG scan demonstrates a favorable response at 6 months. Row B demonstrates non-FES avid osseous metastases which are FDG avid, but does not demonstrate a favorable response to endocrine therapy with progression of hypermetabolic osseous metastases noted on FDG post-treatment scan at 6 months. From Linden HM, Stekhova SA, Link JM, et al. Quantitative fluoroestradiol positron emission tomography imaging predicts response to endocrine treatment in breast cancer. J Clin Oncol. 2006;24:2793-2799, with permission.

Figure 2: FDG-PET/CT compared to ${ }^{89} \mathrm{Zr}$-trastuzumab-PET/CT (HER2-PET/CT) MIP images in HER2+ breast cancer patients. (A) Example of similar distribution of metastases on FDG PET and HER2 PET. (B) The dominant portions of the tumor demonstrate uptake on HER2 PET noting that many of the lung lesions are not seen. (C) The majority of the tumor burden does not demonstrate uptake on HER2-PET/CT. (D) None of the tumor demonstrated uptake on the HER2-PET/CT. From Gebhart G, Lamberts LE, Wimana Z, et al. Molecular imaging as a tool to investigate heterogeneity of advanced HER2-positive breast cancer and to predict patient outcome under trastuzumab emtansine (T-DM1): the ZEPHIR trial. Ann Oncol. 2016;27:619-624, with permission.

Figure 3: FLT-PET/CT in a patient undergoing neoadjuvant treatment. The pretherapy scan in column FLT1 (left) demonstrates FLT uptake in primary mass in the left breast and left axillary lymph node. Column FLT2 (middle) demonstrates decreased tracer uptake after one cycle of neoadjuvant chemotherapy and column FLT3 (right) demonstrates complete metabolic response after completion of neoadjuvant chemotherapy. Pathologic 
complete response was confirmed surgically. From Kostakoglu L, Duan F, Idowu MO, et al. A Phase II Study of 3'-Deoxy-3'-18F-Fluorothymidine PET in the Assessment of Early Response of Breast Cancer to Neoadjuvant Chemotherapy: Results from ACRIN 6688. J Nucl Med. 2015;56(11):1681-1689, with permission.

Figure 4: FDG-PET/CT used for assessing response in two patients with osseous dominant metastatic breast cancer. MIP whole body FDG-PET on top row and fused FDG-PET/CT on bottom row. Panel (A) demonstrating patient on tamoxifen without definite osseous metastases. Follow-up FDG-PET/CT performed 8 months later after switching to aromatase inhibitor (Letrozole) demonstrates new foci of FDG osseous uptake, reflecting progressive metabolic disease (arrows). Panel (B) demonstrating patient with ER+PR+/HER2metastatic breast cancer with innumerable osseous metastases throughout axial and appendicular skeleton (arrows). Follow-up FDG-PET/CT performed after switching treatment to Capecitabine demonstrates significant partial metabolic response with near complete resolution of the majority of osseous lesions with a few scattered foci of FDG uptake reflecting residual disease (arrow).

\section{References:}

1. Siegel RL, Miller KD, Jemal A. Cancer statistics, 2015. CA Cancer J Clin. 65:5-29.

2. Henry NL, Hayes DF. Cancer biomarkers. Mol Oncol. 2012;6:140-146.

3. Hammond MEH, Hayes DF, Wolff AC, Mangu PB, Temin S. American society of clinical oncology/college of american pathologists guideline recommendations for 
immunohistochemical testing of estrogen and progesterone receptors in breast cancer. J Oncol Pract. 2010;6:195-197.

4. Gerlinger M, Rowan AJ, Horswell S, et al. Intratumor heterogeneity and branched evolution revealed by multiregion sequencing. N Engl J Med. 2012;366:883-892.

5. Amir E, Miller N, Geddie W, et al. Prospective study evaluating the impact of tissue confirmation of metastatic disease in patients with breast cancer. J Clin Oncol. $2012 ; 30: 587-592$.

6. Ulaner GA, Riedl CC, Dickler MN, Jhaveri K, Pandit-Taskar N, Weber W. Molecular Imaging of Biomarkers in Breast Cancer. J Nucl Med. 2016;57 Suppl 1:53S - 9S.

7. Fowler AM, Clark AS, Katzenellenbogen JA, Linden HM, Dehdashti F. Imaging Diagnostic and Therapeutic Targets: Steroid Receptors in Breast Cancer. J Nucl Med. 2016;57 Suppl 1:75S - 80S.

8. Gebhart G, Flamen P, De Vries EGE, Jhaveri K, Wimana Z. Imaging Diagnostic and Therapeutic Targets: Human Epidermal Growth Factor Receptor 2. J Nucl Med. 2016;57 Suppl 1:81S - 8S.

9. Gradishar WJ, Anderson BO, Balassanian R, et al. Invasive Breast Cancer Version 1.2016, NCCN Clinical Practice Guidelines in Oncology. J Natl Compr Canc Netw. 2016;14:324-354.

10. Mintun MA, Welch MJ, Siegel BA, et al. Prognostic impact of (18)FDG-PET-CT findings in clinical stage III and IIB breast cancer. Radiology. 1988;169:45-48.

11. Mintun MA, Welch MJ, Siegel BA, et al. ${ }^{18} \mathrm{~F}-\mathrm{FDG}$ PET/CT provides powerful prognostic stratification in the primary staging of large breast cancer when compared with conventional explorations. Radiology. 1988;169:45-48. 
12. Koolen BB, Vrancken Peeters MJTFD, Wesseling J, et al. Association of primary tumour FDG uptake with clinical, histopathological and molecular characteristics in breast cancer patients scheduled for neoadjuvant chemotherapy. Eur J Nucl Med Mol Imaging. 2012;39:1830-1838.

13. Oshida M, Uno K, Suzuki M, et al. Predicting the prognoses of breast carcinoma patients with positron emission tomography using 2-deoxy-2-fluoro[18F]-D-glucose. Cancer. 1998;82:2227-2234.

14. Groheux D, Giacchetti S, Moretti J-L, et al. Correlation of high 18F-FDG uptake to clinical, pathological and biological prognostic factors in breast cancer. Eur J Nucl Med Mol Imaging. 2011;38:426-435.

15. Gil-Rendo A, Martínez-Regueira F, Zornoza G, García-Velloso MJ, Beorlegui C, Rodriguez-Spiteri N. Association between [18F]fluorodeoxyglucose uptake and prognostic parameters in breast cancer. Br J Surg. 2009;96:166-170.

16. Basu S, Chen W, Tchou J, et al. Comparison of triple-negative and estrogen receptorpositive/progesterone receptor-positive/HER2-negative breast carcinoma using quantitative fluorine-18 fluorodeoxyglucose/positron emission tomography imaging parameters: a potentially useful method for d. Cancer. 2008;112:995-1000.

17. Bos R, van Der Hoeven JJM, van Der Wall E, et al. Biologic correlates of (18)fluorodeoxyglucose uptake in human breast cancer measured by positron emission tomography. J Clin Oncol. 2002;20:379-387.

18. Avril S, Muzic RF, Plecha D, Traughber BJ, Vinayak S, Avril N. 18F-FDG PET/CT for Monitoring of Treatment Response in Breast Cancer. J Nucl Med. 2016;57 Suppl $1: 34 S-9 S$. 
19. Osborne CK, Schiff R. Mechanisms of endocrine resistance in breast cancer. Annu Rev Med. 2011;62:233-247.

20. Peterson LM, Mankoff DA, Lawton T, et al. Quantitative imaging of estrogen receptor expression in breast cancer with PET and 18F-fluoroestradiol. J Nucl Med. 2008;49:367-374.

21. Mintun MA, Welch MJ, Siegel BA, et al. Breast cancer: PET imaging of estrogen receptors. Radiology. 1988;169:45-48.

22. Dehdashti F, Flanagan FL, Mortimer JE, Katzenellenbogen JA, Welch MJ, Siegel BA. Positron emission tomographic assessment of "metabolic flare" to predict response of metastatic breast cancer to antiestrogen therapy. Eur J Nucl Med. 1999;26:51-56.

23. Dehdashti F, Mortimer JE, Trinkaus K, et al. PET-based estradiol challenge as a predictive biomarker of response to endocrine therapy in women with estrogenreceptor-positive breast cancer. Breast Cancer Res Treat. 2009;113:509-517.

24. Linden HM, Stekhova SA, Link JM, et al. Quantitative fluoroestradiol positron emission tomography imaging predicts response to endocrine treatment in breast cancer. J Clin Oncol. 2006;24:2793-2799.

25. Mortimer JE, Dehdashti F, Siegel BA, Trinkaus K, Katzenellenbogen JA, Welch MJ. Metabolic flare: indicator of hormone responsiveness in advanced breast cancer. J Clin Oncol. 2001;19:2797-2803.

26. van Kruchten M, de Vries EGE, Brown M, et al. PET imaging of oestrogen receptors in patients with breast cancer. Lancet Oncol. 2013;14:e465-e475.

27. Dehdashti F. [18F] Fluoroestradiol (FES) PET as a Predictive Measure for Endocrine Therapy in Patients With Newly Diagnosed Metastatic Breast Cancer. 
ClinicalTrials.gov [Internet]. Bethesda (MD): National Library of Medicine (US). April 2016. Available from: https://clinicaltrials.gov/ct2/show/NCT02398773: NCT02398773

28. Lee JH, Zhou H, Dence CS, Carlson KE, Welch MJ, Katzenellenbogen JA. Development of [F-18]fluorine-substituted Tanaproget as a progesterone receptor imaging agent for positron emission tomography. Bioconjug Chem. 2010;21:1096-1104.

29. Dehdashti F, Laforest R, Gao F, et al. Assessment of progesterone receptors in breast carcinoma by PET with 21-18F-fluoro-16 $\alpha, 17 \alpha-[(\mathrm{R})-(1$ '- $\alpha$-furylmethylidene)dioxy]19-norpregn-4-ene-3,20-dione. J Nucl Med. 2012;53:363-370.

30. Fowler AM, Chan SR, Sharp TL, et al. Small-animal PET of steroid hormone receptors predicts tumor response to endocrine therapy using a preclinical model of breast cancer. J Nucl Med. 2012;53:1119-1126.

31. Dehdashti F. Assessment of Functional Status of Estrogen Receptors in Breast Cancer by Positron Emission Tomography. In: ClinicalTrials.gov [Internet]. Bethesda (MD): National Library of Medicine (US). April 2016. Available from: https://clinicaltrials.gov/ct2/show/NCT02455453: NCT02455453.

32. Ross JS, Fletcher JA. The HER-2/neu oncogene in breast cancer: prognostic factor, predictive factor, and target for therapy. Stem Cells. 1998;16:413-428.

33. Sjögren S, Inganäs M, Lindgren A, Holmberg L, Bergh J. Prognostic and predictive value of c-erbB-2 overexpression in primary breast cancer, alone and in combination with other prognostic markers. J Clin Oncol. 1998;16:462-469.

34. Slamon DJ, Clark GM, Wong SG, Levin WJ, Ullrich A, McGuire WL. Human breast cancer: correlation of relapse and survival with amplification of the HER-2/neu 
oncogene. Science. 1987;235:177-182.

35. Slamon DJ, Godolphin W, Jones LA, et al. Studies of the HER-2/neu proto-oncogene in human breast and ovarian cancer. Science. 1989;244:707-712.

36. Slamon DJ, Leyland-Jones B, Shak S, et al. Use of Chemotherapy plus a Monoclonal Antibody against HER2 for Metastatic Breast Cancer That Overexpresses HER2. N Engl J Med. 2001;344:783-792.

37. Gebhart G, Gámez C, Holmes E, et al. 18F-FDG PET/CT for early prediction of response to neoadjuvant lapatinib, trastuzumab, and their combination in HER2positive breast cancer: results from Neo-ALTTO. J Nucl Med. 2013;54:1862-1868.

38. Potts SJ, Krueger JS, Landis ND, et al. Evaluating tumor heterogeneity in immunohistochemistry-stained breast cancer tissue. Lab Investig. 2012;92:13421357.

39. Mankoff DA, Link JM, Linden HM, Sundararajan L, Krohn KA. Tumor receptor imaging. J Nucl Med. 2008;49 Suppl 2:149S - 63S.

40. Linden HM, Dehdashti F. Novel methods and tracers for breast cancer imaging. Semin Nucl Med. 2013;43:324-329.

41. Gebhart G, Lamberts LE, Wimana Z, et al. Molecular imaging as a tool to investigate heterogeneity of advanced HER2-positive breast cancer and to predict patient outcome under trastuzumab emtansine (T-DM1): the ZEPHIR trial. Ann Oncol. 2016;27:619-624.

42. Flamen P. Pilot Imaging Study With 89Zr-Trastuzumab in HER2-positive Metastatic Breast Cancer Patients: Correlation With FDG-PET/CT and Anatomopathological Results. In: ClinicalTrials.gov [Internet]. Bethesda (MD): National Library of Medicine 
(US). April 2016. Available from: https://clinicaltrials.gov/ct2/show/NCT01420146: NCT01420146.

43. Schroder C. P. HER2-PET as a Diagnostic Tool in Breast Cancer Patients With a Clinical Dilemma. In: ClinicalTrials.gov [Internet]. Bethesda (MD): National Library of Medicine (US). April 2016. Available from: https://clinicaltrials.gov/ct2/show/NCT01832051: NCT01832051.

44. Schroder C.P. Towards Patient Tailored Cancer Treatment Supported by Molecular Imaging IMPACT: IMaging PAtients for Cancer Drug selecTion - Metastatic Breast Cancer. In: ClinicalTrials.gov [Internet]. Bethesda (MD): National Library of Medicine (US). April 2016. Available from: https://clinicaltrials.gov/ct2/show/NCT01957332: NCT01957332.

45. Linden HM, Kurland BF, Peterson LM, et al. Fluoroestradiol positron emission tomography reveals differences in pharmacodynamics of aromatase inhibitors, tamoxifen, and fulvestrant in patients with metastatic breast cancer. Clin Cancer Res. 2011;17:4799-4805.

46. Robertson JFR, Llombart-Cussac A, Rolski J, et al. Activity of fulvestrant $500 \mathrm{mg}$ versus anastrozole $1 \mathrm{mg}$ as first-line treatment for advanced breast cancer: results from the FIRST study. J Clin Oncol. 2009;27:4530-4535.

47. Di Leo A, Jerusalem G, Petruzelka L, et al. Results of the CONFIRM phase III trial comparing fulvestrant $250 \mathrm{mg}$ with fulvestrant $500 \mathrm{mg}$ in postmenopausal women with estrogen receptor-positive advanced breast cancer. J Clin Oncol. 2010;28:45944600.

48. van Kruchten M, de Vries EG, Glaudemans AW, et al. Measuring residual estrogen 
receptor availability during fulvestrant therapy in patients with metastatic breast cancer. Cancer Discov. 2015;5:72-81.

49. Heidari P, Deng F, Esfahani SA, et al. Pharmacodynamic imaging guides dosing of a selective estrogen receptor degrader. Clin Cancer Res. 2015;21:1340-1347.

50. Wang, Yingbing; Ulaner, Gary; Manning, Charles; Bardia, Aditya; Mayer, Ingrid; Dickler, Maura; Maneval, Edna Chow; Lewis, Jason; Baselga, Jose; Mahmood U. Validation of target engagement using 18F-fluoroestradiol PET in patients undergoing therapy with selective estrogen receptor degrader, ARN-810 (GDC0810). J Nucl Med. 2015;56:565.

51. Lai A, Kahraman M, Govek S, et al. Identification of GDC-0810 (ARN-810), an Orally Bioavailable Selective Estrogen Receptor Degrader (SERD) that Demonstrates Robust Activity in Tamoxifen-Resistant Breast Cancer Xenografts. J Med Chem. 2015;58:4888-4904.

52. Gaykema SBM, Schröder CP, Vitfell-Rasmussen J, et al. 89Zr-trastuzumab and 89Zrbevacizumab PET to evaluate the effect of the HSP90 inhibitor NVP-AUY922 in metastatic breast cancer patients. Clin Cancer Res. 2014;20:3945-3954.

53. Kurland BF, Gadi VK, Specht JM, et al. Feasibility study of FDG PET as an indicator of early response to aromatase inhibitors and trastuzumab in a heterogeneous group of breast cancer patients. EJNMMI Res. 2012;2:34.

54. Kenny L. The Use of Novel PET Tracers to Image Breast Cancer Biologic Processes Such as Proliferation, DNA Damage and Repair, and Angiogenesis. J Nucl Med. 2016;57 Suppl 1:89S - 95S.

55. Bading JR, Shields AF. Imaging of cell proliferation: status and prospects. J Nucl Med. 
2008;49 Suppl 2:64S - 80S.

56. Kenny LM, Vigushin DM, Al-Nahhas A, et al. Quantification of cellular proliferation in tumor and normal tissues of patients with breast cancer by [18F]fluorothymidinepositron emission tomography imaging: evaluation of analytical methods. Cancer Res. 2005;65:10104-10112.

57. Woolf DK, Beresford M, Li SP, et al. Evaluation of FLT-PET-CT as an imaging biomarker of proliferation in primary breast cancer. Br J Cancer. 2014;110:28472854.

58. Dowsett M, Archer C, Assersohn L, et al. Clinical studies of apoptosis and proliferation in breast cancer. Endocr Relat Cancer. 1999;6:25-28.

59. Jones RL, Salter J, A'Hern R, et al. The prognostic significance of Ki67 before and after neoadjuvant chemotherapy in breast cancer. Breast Cancer Res Treat. 2009;116:5368.

60. Kenny L, Coombes RC, Vigushin DM, Al-Nahhas A, Shousha S, Aboagye EO. Imaging early changes in proliferation at 1 week post chemotherapy: a pilot study in breast cancer patients with 3'-deoxy-3'-[18F]fluorothymidine positron emission tomography. Eur J Nucl Med Mol Imaging. 2007;34:1339-1347.

61. Contractor KB, Kenny LM, Stebbing J, et al. [18F]-3’Deoxy-3'-fluorothymidine positron emission tomography and breast cancer response to docetaxel. Clin Cancer Res. 2011;17:7664-7672.

62. Kostakoglu L, Duan F, Idowu MO, et al. A Phase II Study of 3'-Deoxy-3'-18FFluorothymidine PET in the Assessment of Early Response of Breast Cancer to Neoadjuvant Chemotherapy: Results from ACRIN 6688. J Nucl Med. 2015;56:1681- 
1689.

63. Kenny LM, Contractor KB, Stebbing J, et al. Altered tissue 3'-deoxy-3'[18F]fluorothymidine pharmacokinetics in human breast cancer following capecitabine treatment detected by positron emission tomography. Clin Cancer Res. 2009;15:6649-6657.

64. Wheeler KT, Wang LM, Wallen CA, et al. Sigma-2 receptors as a biomarker of proliferation in solid tumours. Br J Cancer. 2000;82:1223-1232.

65. Mach RH, Smith CR, Al-Nabulsi I, Whirrett BR, Childers SR, Wheeler KT. Sigma 2 receptors as potential biomarkers of proliferation in breast cancer. Cancer Res. $1997 ; 57: 156-161$.

66. Vilner BJ, John CS, Bowen WD. Sigma-1 and sigma-2 receptors are expressed in a wide variety of human and rodent tumor cell lines. Cancer Res. 1995;55:408-413.

67. Dehdashti F, Laforest R, Gao F, et al. Assessment of cellular proliferation in tumors by PET using 18F-ISO-1. J Nucl Med. 2013;54:350-357.

68. Shoghi KI, Xu J, Su Y, et al. Quantitative receptor-based imaging of tumor proliferation with the sigma-2 ligand [(18)F]ISO-1. PLoS One. 2013;8:e74188.

69. McDonald ES, Mankoff DA, Mach RH. Novel Strategies for Breast Cancer Imaging: New Imaging Agents to Guide Treatment. J Nucl Med. 2016;57 Suppl 1:69S - 74S.

70. Kenny LM, Contractor KB, Hinz R, et al. Reproducibility of [11C]choline-positron emission tomography and effect of trastuzumab. Clin Cancer Res. 2010;16:42364245.

71. Huovinen R, Leskinen-Kallio S, Någren K, Lehikoinen P, Ruotsalainen U, Teräs M. Carbon-11-methionine and PET in evaluation of treatment response of breast cancer. 
Br J Cancer. 1993;67:787-791.

72. Jansson T, Westlin JE, Ahlström H, Lilja A, Långström B, Bergh J. Positron emission tomography studies in patients with locally advanced and/or metastatic breast cancer: a method for early therapy evaluation? J Clin Oncol. 1995;13:1470-1477.

73. Lindholm P, Lapela M, Någren K, Lehikoinen P, Minn H, Jyrkkiö S. Preliminary study of carbon-11 methionine PET in the evaluation of early response to therapy in advanced breast cancer. Nucl Med Commun. 2009;30:30-36.

74. Hamaoka T, Madewell JE, Podoloff DA, Hortobagyi GN, Ueno NT. Bone imaging in metastatic breast cancer. J Clin Oncol. 2004;22:2942-2953.

75. Morris PG, Ulaner GA, Eaton A, et al. Standardized uptake value by positron emission tomography/computed tomography as a prognostic variable in metastatic breast cancer. Cancer. 2012;118:5454-5462.

76. Specht JM, Tam SL, Kurland BF, et al. Serial 2-[18F] fluoro-2-deoxy-D-glucose positron emission tomography (FDG-PET) to monitor treatment of bone-dominant metastatic breast cancer predicts time to progression (TTP). Breast Cancer Res Treat. 2007;105:87-94.

77. Tateishi U, Gamez C, Dawood S, Yeung HWD, Cristofanilli M, Macapinlac HA. Bone metastases in patients with metastatic breast cancer: morphologic and metabolic monitoring of response to systemic therapy with integrated PET/CT. Radiology. 2008;247:189-196.

78. Du Y, Cullum I, Illidge TM, Ell PJ. Fusion of metabolic function and morphology: sequential [18F]fluorodeoxyglucose positron-emission tomography/computed tomography studies yield new insights into the natural history of bone metastases in 
breast cancer. J Clin Oncol. 2007;25:3440-3447.

79. Emmering J, Krak NC, Van der Hoeven JJM, et al. Preoperative [18F] FDG-PET after chemotherapy in locally advanced breast cancer: prognostic value as compared with histopathology. Ann Oncol. 2008;19:1573-1577.

80. Dunnwald LK, Doot RK, Specht JM, et al. PET tumor metabolism in locally advanced breast cancer patients undergoing neoadjuvant chemotherapy: value of static versus kinetic measures of fluorodeoxyglucose uptake. Clin Cancer Res. 2011;17:24002409.

81. Dunnwald LK, Gralow JR, Ellis GK, et al. Tumor metabolism and blood flow changes by positron emission tomography: relation to survival in patients treated with neoadjuvant chemotherapy for locally advanced breast cancer. J Clin Oncol. 2008;26:4449-4457.

82. Dunnwald LK, Gralow JR, Ellis GK, et al. Residual tumor uptake of [99mTc]-sestamibi after neoadjuvant chemotherapy for locally advanced breast carcinoma predicts survival. Cancer. 2005;103:680-688. 


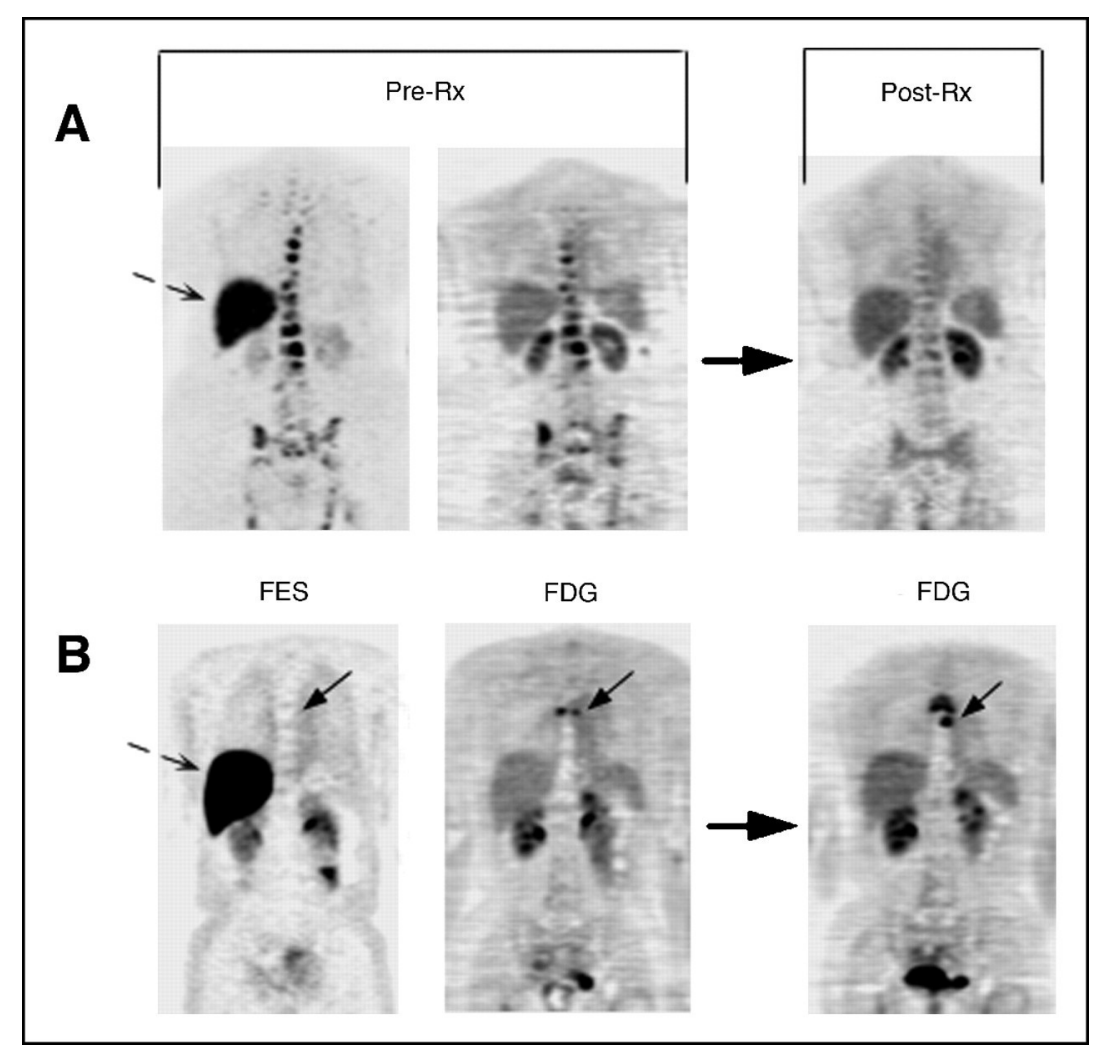




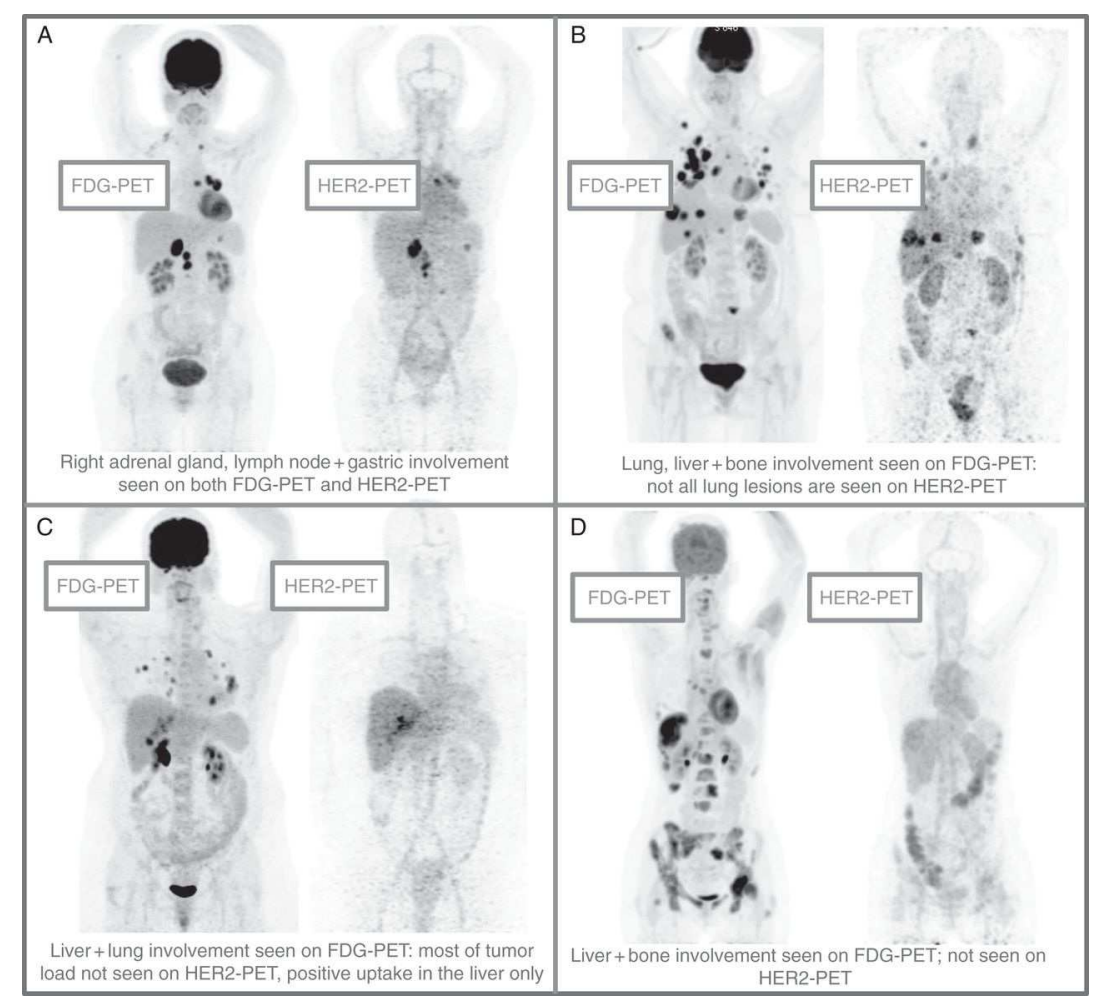


FLT 1
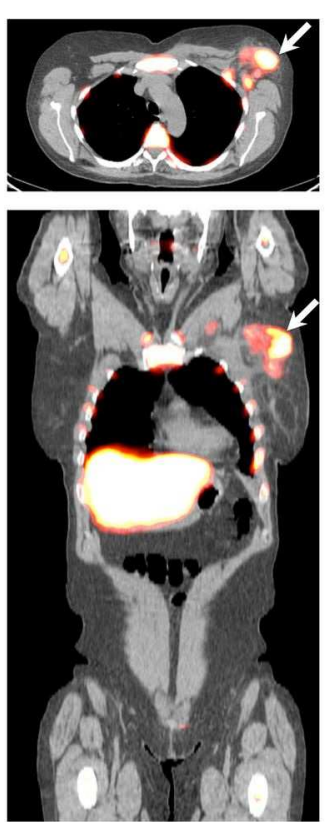

FLT SUVmax $=7.79$ Ki-67 = 70\%
FLT 2
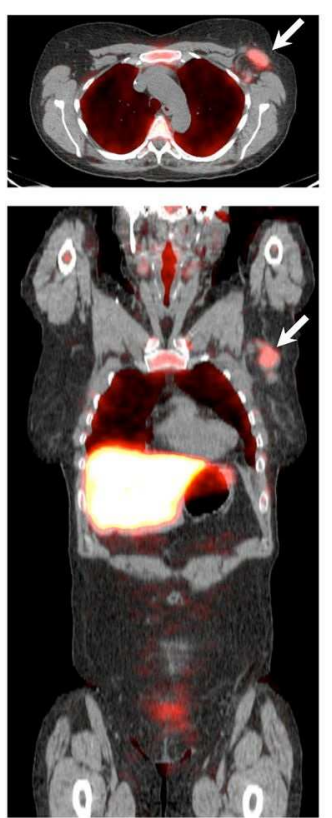

FLT SUVmax $=2.93$
Ki-67 = No Sample

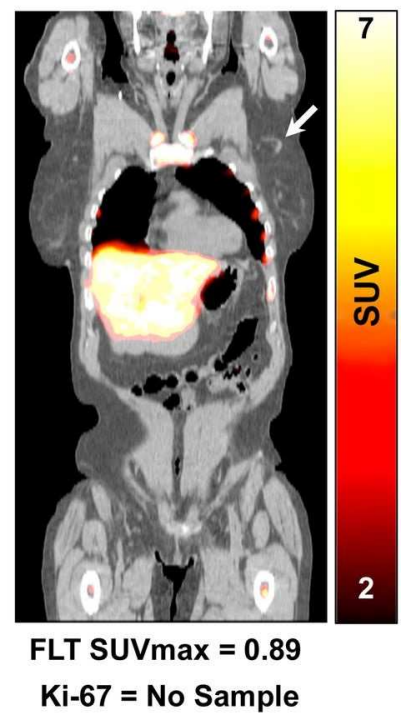

2

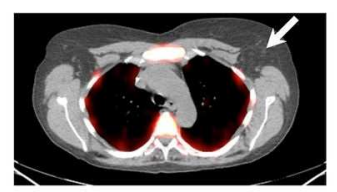




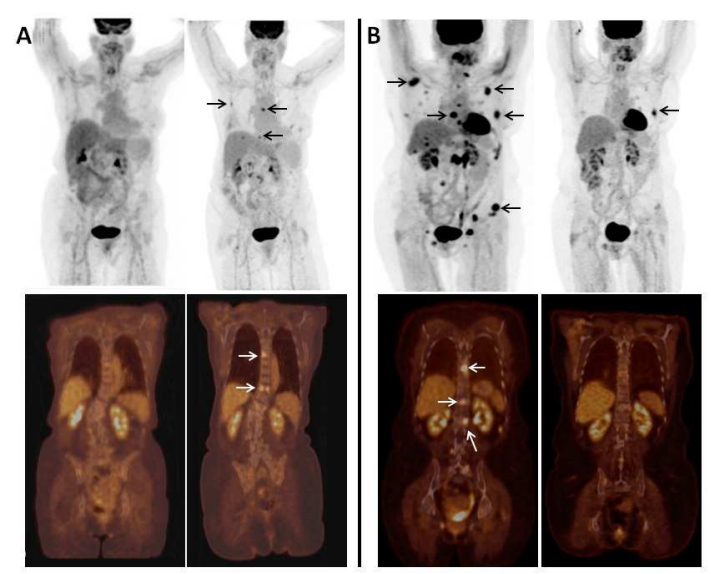

